\title{
Research on the Wireless sensor Network Data Fusion Technology
}

\author{
Ya-qin Fan \\ College of Communication. \\ Engineering, Jilin University, \\ Changchun ,130012, china \\ E-mail: Zhangnan9970@126.com
}

\author{
Yu Ding \\ Public Sercurity Bureau of \\ Changchun City , \\ Changchun ,130051, china \\ E-mail:dingsunyuli@163.com
}

\author{
Mei-lin Liu, Xin Zhang \\ College of Communication. \\ Engineering, Jilin University, \\ Changchun ,130012, china \\ E-mail: 940614745@qq.com
}

\begin{abstract}
Wireless sensor network ( WSN ) is a kind of energy constrained network, by using data fusion technology, the elimination of redundant data, can save energy, prolong the network life purpose. Data fusion in wireless sensor network can realize different protocol layers, Based on the introduction of wireless sensor network and data fusion related knowledge, prove that the arithmetic mean method is effective, and use OPNET software tool for network simulation, finally, analysis results and conclude, verify effects of the arithmetic average fusion algorithm for wireless sensor network.
\end{abstract}

Key words-WSN; Data fusion; Average method; OPNET

\section{OVERVIEW}

Wireless sensor network is increasingly paid attention extensively, but its energy Limited is the development, has become a hot research issue. In order to improve the energy utilization rate, many scholars of the data fusion technology of wireless sensor networks with effective combination, the transmission of data and node energy consumption than less energy consumption is corresponding to the data processing, data fusion is used to increase the wireless sensor network data processing to reduce network data transmission, in order to reduce the energy consumption of the whole network, to extend its life cycle.

\section{WIRELESS SENSOR NETWORK STRUCTURE}

Sensor nodes of wireless sensor network, from the network topology structure, according to the size of the nodes of wireless sensor networks structure is divided into the plane structure and hierarchical structure of two. When the network of small scale, less nodes, using a planar structure, such as a number of small household sensor network; and when the network is larger, the number of nodes is various, must use the hierarchical structure.

\section{A. Plane Structure}

As shown in Figure 1, in the flat structure nodes equal in status, for peer-to-peer structure.

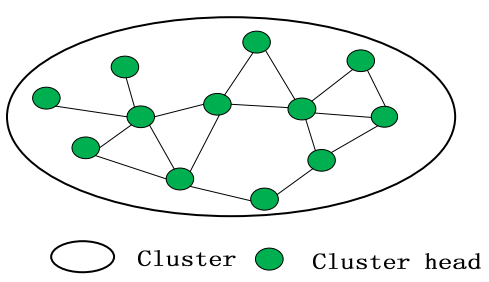

Fig 1 Schematic diagram of planar structure

\section{B. Layered Structure}

Fig 2 Schematic diagram for hierarchical structure, the entire network from a plurality of clusters, each cluster includes a cluster head and a plurality of the cluster members, cluster head constitute high level network.

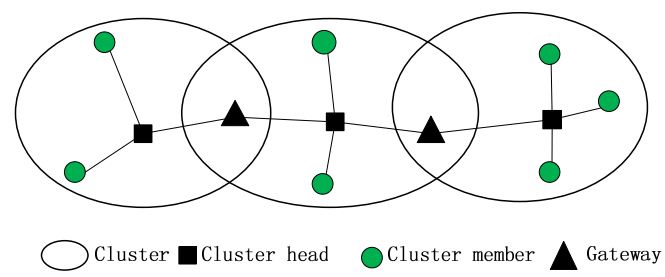

Fig 2 Schematic diagram of hierarchical structure

\section{ARCHITECTURE OF WIRELESS SENSOR NETWORKS}

Wireless sensor network target for application oriented network information source, through infrared, radar or sonar, seismic signal, to obtain include temperature, noise and motion direction and speed of the target attribute, used to achieve a predetermined objective. As mentioned before, wireless sensor network architecture is composed of wireless sensor network and gateway, external transmission network, base station, the composition such as the user, figure 3 is its schematic diagram. 


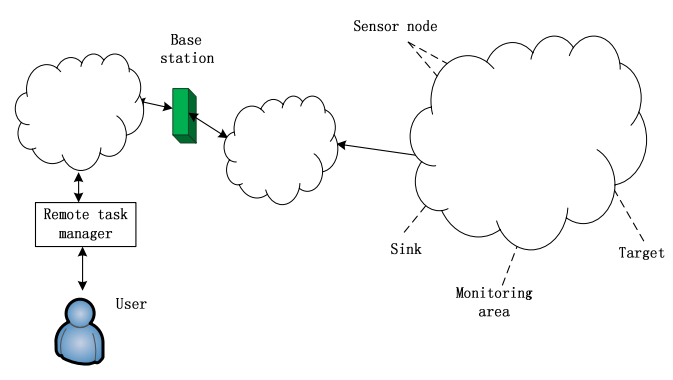

Figure 3 the architecture of Wireless Sensor Networks

\section{THE CONCEPT OF DATA FUSION}

Data Fusion, also known as information fusion, its research scope is inconclusive, the earliest is applied in the military field, according to the field application, data fusion can be defined as: the spatial distribution of the various sensors on the spatial-temporal sampling, target detection, tracking and estimation, interconnection, combination of multi-source information and data multiple layer, multiple aspects of processing, in order to obtain accurate state estimation and identity, as well as higher levels of complete and timely tactical situation and threat assessment[1].

\section{WSN DATA FUSION SIMULATION OPNET}

In this paper, using network simulation tool for OPNET software, the specific use is OPNET11.5 version. OPNET provides three layer modeling mechanism, from the ground about respectively: process modeling, node model and network model, the network model to set up a Project to contain the Scenario.

In this paper, the wireless sensor network node is set to a 13 node, each node equal status, network structure to plane structure, for the sake of good looking, 13 nodes network topology map in a tree form, as shown in Figure 4 below.

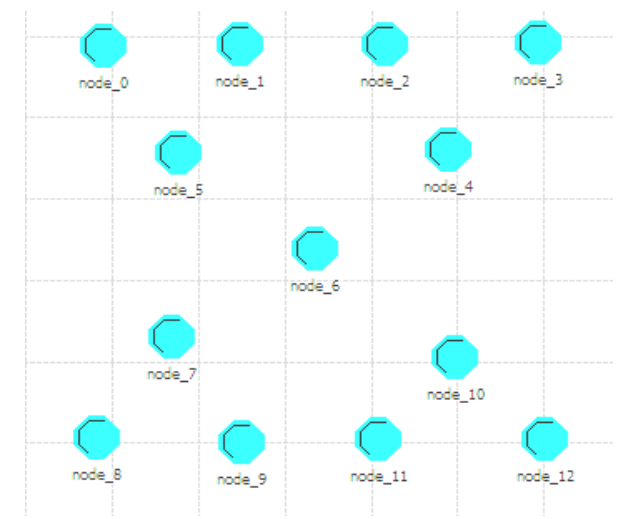

Figure 4 wireless sensor network topology
The OPNET three layer modeling are involved, the following will introduce based on the arithmetic of wireless sensor network data fusion specific modeling and simulation result output procedure.

\section{A. OPNET Modeling}

As everyone knows, the network communication process consists of the terminal and link, link is the transmission signal media, divided into the cable channel and the radio channel. Wireless sensor network channel for wireless channel, is required in the transceiver configured to channel, usually can be arranged as required multiple channels. Therefore for modeling wireless sensor networks including Packet, Node, Process and Net, wireless channel allocation in joint modeling of transceiver to add the desired channel, antenna used for the omnidirectional antenna.

Wireless sensor networks OPNET modeling is divided into the following three steps: create data packet node model; modeling; network modeling.

1) Create packets model

a) From the File menu option from the drop-down menu in the New, found in the Packet Format and open;

b) Click on the Create New Field button, the data frame into the editor editor window, select the data box, click the right mouse button, select the Edit Attributes size, set the length of data packet, but also in the color item to choose their own like colors, and named for its own easy recognition name[2-3];

c) Click the OK, at this point in the editor window will display the edited data packet format.

Last saved ( Save ) packets, named packet_WSN. As a result of this is a OPNET inside its own packet format, so the above is only used by the packet creation process, by the use of the packet too big in the thesis will be no graphics display.

2) Node model

The node model, using OPNET in the original wlan_station_adv ( fix ) model, and some slight modifications and in the process modeling in data fusion are.

a) From the File New, in New from the drop-down menu to select Node Model and open;

b) Click the Create Processor button, drag node editing window, then continuing into the three process block, and from lower to upper drain three layer, the first layer is a layer second process module, a process module, third layer parallel to put two of the process modules [4].

c) Click the bottom p_0, and then click the right mouse button, select Edit Attributes process model, Value wlan_dispatch, and the name into wireless_lan_mac, and some attributes to attribute node, the status is set to promoted;

d) In the same way to open the second layer $p_{-} 1$, process model Value wlan_mac_interface, name to 
wlan_mac_intf, can also be part of property promotion for node property, convenient in later in the environment of the network set up and modify, and the third layer of the two process block p_2 and p_3 name to source and sink. ;

e) To the top of the source and sink for data generation and reception, source process model is set to bursty_source, sink is set to sink, and the source Packet Interarrivl time attribute which is promoted to promoted, and its name is renamed to Interarrivl time[5-6];

f) In Interfaces Node Interfaces, Attributes Interarrivl time found in Edit, click Properties, click to enter, in Symbol map all Status was changed to suppress, and adding several symbols and values in the mapping table, respectively 5, 10, 50 and 100, see figure 5 ;

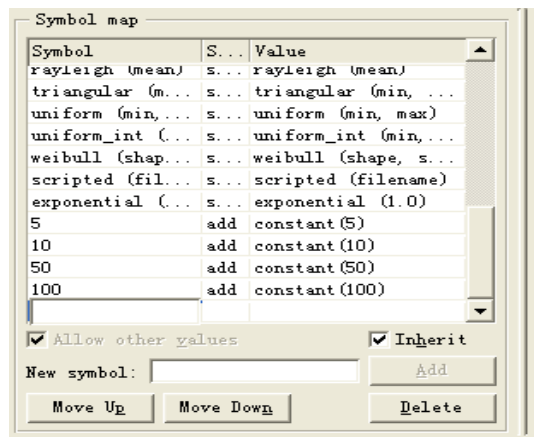

Fig 5 symbols and attributes of the real value of the mapping table

g) Click on the Create Packet Stream button, the process block with the packet flow (Create Packet Stream ) is correctly connected, in order to check the connection is correct, you can click to view the process blocks, click the right mouse button to select Show Conectivity view the packet flow allocation table. In order to distinguish the packet stream input and output, send and receive packet stream with red and blue in two different colors, and the transceiver and antenna (see Figure 6 ). Among them, the omnidirectional antenna is click on the Create Antenna button, the Attributes inside the pattern option for isotropic, in the antenna three-dimensional graphics for a uniform ball;

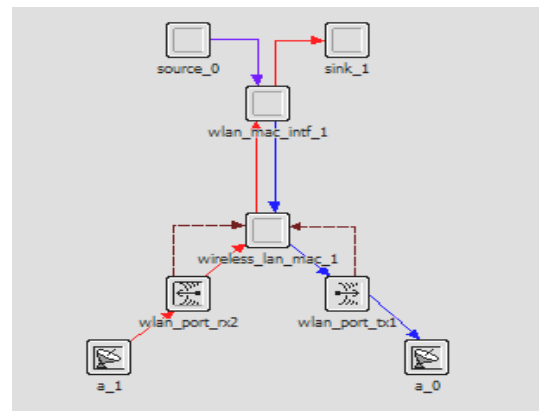

Figure 6 complete node node model h) From the Interfaces Interfaces Node type Node, in satellite Supported will be changed to no, it follows that node types can be fixed node (fixed) and the mobile node ( mobile ), rather than a satellite node, click on the OK back to the editor;

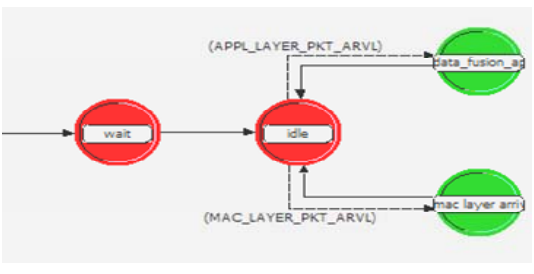

Fig 7 part wlan_mac_intf_1 process model

Last saved ( Save ) node and the node named node, close the node editor. The same process to create data fusion network nodes fusion_node, adding the data fusion arithmetic average, a key part of the process model pictured above 7 .

3) Network modeling

a) In the OPNET Modeler main window, select the File, selecting from the drop-down menu in the menu New, select Project, click OK;

b) In the pop-up dialog box, Project name named undata_fusion Scenario name, named WSN, click OK, and step by step press has been in accordance with Next, but in the network scale selection at the office, and is set to 100 for $X, 100$ for $y$, meter $100 \mathrm{~m} 100 \mathrm{~m}$ unit network range, click on the Finish after entering the project editor window;

c) Open object panel (Object Palette), click the Search by name Object Palette tree button from the left into the Object Palette interface, click on the right side of the Configure Palette in the Configure Palette interface; click on Clear, then click on the Node Model, find the first created the node nodes and data_node, Status to included, click $\mathrm{OK}$, and then click on the Configure Palette interface OK, save it to a WSN_palette, see figure 8;

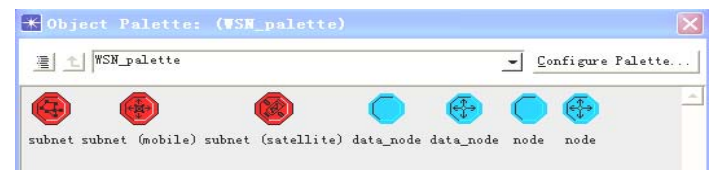

Figure 8 WSN_palette objects panel

d) 13 node node to drag the project editor, according to 4-1 network topology construction of network engineering, will save. The above is not the network data fusion, data fusion networks using the same scene WSN, this project will be saved as a data_fusion, in the data_fusion project, hold down the Ctrl key to select all the nodes in the node attributes, in Attributes process model to fusion_node[7-10]. 


\section{B. Collection of Variable and Result Output}

In order to distinguish the settings of different Interarrivl two of time group network, project data_fusion and undata_fusion respectively corresponding to the engineering zzd1 and zzd2, the scene is always WSN. Simulation in the laboratory after the operation, view the results, four intervals of the rules are the same, but the effect is better for both 5 and 50 , this election will use them for this experiment configuration data.

1) Set up the project data_fusion and undata_fusion all nodes of the Attributes Interarrivl time 5, respectively, on the collection of variables. Specific view of each network throughput, as shown in figure 9.

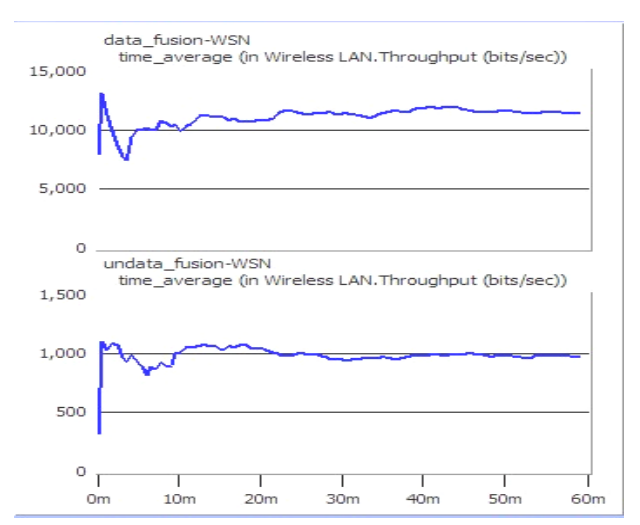

Fig 9 Comparison of the throughput of the network respectively display

2) Set up the project zzd1-WSN and zzd2-WSN all nodes of the Attributes Interarrivl time 50, separately carries on the variables collected. Specific view of each network throughput, as shown in figure10.

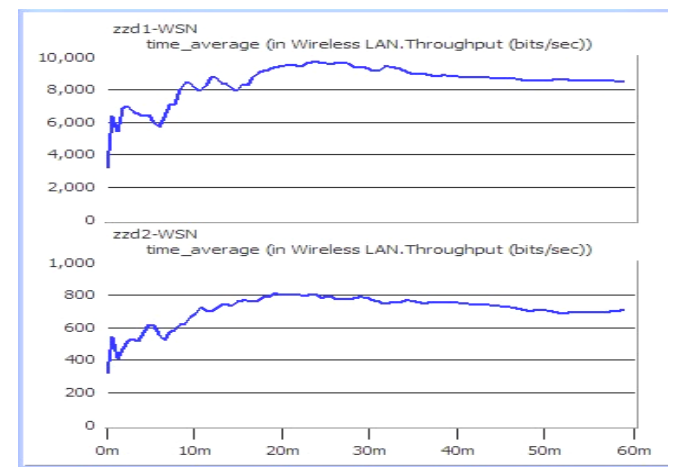

Fig 10 Comparison of the throughput of the network respectively display

\section{The Results of Analysis}

The packet arrival time interval of 5 , figure 5 direct derived data fusion in wireless sensor network data fusion in wireless sensor network is not the network throughput. Packet arrival time interval of 50, Figure 6 can be seen clearly after data fusion for wireless sensor network data fusion than before the network throughput. The two group analysis and draws the conclusion: data fusion network end-to-end delay is increased, but also make the throughput of the network becomes larger. Wireless sensor network for several performance indexes is interconnected between the. Normally, people will be based on specific application needs to improve a performance index and reduced another performance index. In this paper, the simulation results are presented based on take the arithmetic mean of data fusion algorithm based on wireless sensor network availability.

\section{SUMMARY}

As the wireless sensor network to its own characteristics, energy of nodes, bandwidth, processing power, storage capacity and communication bandwidth is limited, and the energy of nodes is limited seriously affect the working life of wireless sensor network. In this case, the data fusion technology is combined with, to delete redundant data, in order to increase the data processing to reduce the data transmission, thereby reducing the energy consumption, prolong the network life. Defects of the paper lies in the construction of network simulation and algorithm is relatively simple. WSN data fusion technology is a most challenging task, in the future to more advanced mesh network as well as more complex Calman filtering method or Bias algorithm!

\section{REFERENCE}

[1] Tang Hong, Xie Jing, Lu Yufang, wireless sensor network theory and application. Beijing: People's post and Telecommunications Publishing House, 2010/8.

[2] [the ]Lawrence A.Klein. Dai Yaping, Liu syndrome, .Sensor and Data Fusion Yu brilliant translation of Concepts and Applications. Beijing: Beijing Institute of Technology press, 2004.129-134

[3] L K rishnamachari, D Estrin, S Wicker.The Impact of Data Aggregation in Wireless Sensor Networks.In:Distributed Computing Systems Workshops, Proceedings of 22nd International Conference.Washington DC:IEEE Computer Society, 2002:575-578

[4] Mohammad Momanni, Subhash Challa, Rami Alhmouz.Bayesian Fusion Algorithm for Inferring Trust in Wireless Sensor Networks.Journal of networks, Vol. 5, No. 7, July 2010

[5] Kang Jian, left charter, Tang Liwei, Zhang Xihong, Li Jie, wireless sensor network data fusion technology [ J] Journal of computer science, 2010/4, thirty-seventh Volume 4 issue.

[6] Fen, wireless sensor and data fusion [ J] Journal of network security technology and application, 2009/11.

[7] ZHANG Wen-sheng, CAO Guo-hong.Group Rekeying for Filtering False Data in Sensor Networks:A Redistribution and Local Collaboration Based Approach[C] .Proc of IEEE INFOCOM[A].2005:56-60

[8] SUW T, CHANGKM, KUOY H.An Energy Efficient Hybrid Intrusion Prohibition System for Cluster Based Wireless Sensor Networks[J].Computer Networks.2007.51 ( 4) :1151-1168

[9] wireless sensor network data fusion research. Master Dissertation of Henan University of Science and Technology, 2009/12.

[10] Yang Mian, wireless sensor network data fusion routing algorithm research. Master Dissertation of Taiyuan University of Technology, 2009/5. 